\title{
Photometric observations of 9 Near-Earth Objects ${ }^{\star}$
}

\author{
Gy. M. Szabó ${ }^{1,4}$, B. Csák ${ }^{2,4}$ K. Sárneczky ${ }^{3,4}$, and L. L. Kiss ${ }^{1,4}$ \\ 1 Department of Experimental Physics \& Astronomical Observatory, University of Szeged, 6720 Szeged, \\ Dóm tér 9, Hungary \\ 2 Department of Optics \& Quantum Electronics \& Astronomical Observatory, University of Szeged, POB 406, \\ 6701 Szeged, Hungary \\ 3 Department of Physical Geography, ELTE University, 1088 Budapest, Ludovika tér 2., Hungary \\ ${ }^{4}$ Guest Observer at Konkoly Observatory
}

Received 13 March 2001 / Accepted 8 June 2001

\begin{abstract}
We present new CCD observations of nine Near-Earth Asteroids carried out between February, 1999 and July, 2000. The bulk of the data was acquired through an $R_{\mathrm{C}}$ filter, while the minor planet 11405 was observed without filter. We could determine synodic periods and amplitudes for 5 asteroids, $699: 3.3,0.18 ; 1866$ : 2. $7,0^{\mathrm{m}} \cdot 12 ; 1999$ JD6: $7.68,1^{\mathrm{m}} \cdot 2 ; 2000$ GK137: 4. $84,0.27 ; 2000$ NM: 9. $24,0.30$. Based on observations taken at different phases, we could infer a phase parameter $m$ of $0.018 \pm 0.005$ for 1865 Cerberus. An epoch-method yielded a sidereal period of $0.27024003(5)$ for this object with retrograde rotation. The remaining 3 objects have only partial coverage, thus no firm conclusion on their synodic period is possible.
\end{abstract}

Key words. solar system - minor planets

\section{Introduction}

The new observing strategies using fully automatic telescopes (e.g. project NEAT, Pravdo et al. 1999, LINEAR, Stokes et al. 2000) and dedicated large instruments led to regular discoveries of a wealth of relatively bright asteroids, including a considerable number of Near-Earth Objects (NEOs). The quickly responding networks of amateur and professional observatories allow an accurate determination of the orbit even for NEOs with high daily motions, avoiding their loss due to the poor astrometry that was typical a few years ago (Steel \& Marsden 1996). Therefore, in solar system studies this is one of the research fields in which most progress is being made. During recent years there have been intensive studies of these objects owing to the recognition of their possible impact hazards (Binzel 2000).

The well-covered NEOs can be studied with photometric methods with great accuracy. Ground-based shape and rotation models require precise time-resolved photometry through several oppositions and this is the main reason why relatively few models in the literature do exist. Possible binarity or precession similarly affect the

Send offprint requests to: L. L. Kiss, e-mail: 1.kiss@physx.u-szeged.hu

* Based on observations taken at the German-Spanish Astronomical Centre, Calar Alto, operated by the Max-PlanckInstitute for Astronomy, Heidelberg, jointly with the Spanish National Commission for Astronomy. lightcurves; therefore, to choose between the possibilities, further high-precision lightcurves are needed (see, e.g., Binzel 1985; Kiss et al. 1999; Pravec et al. 2000).

We started a long-term observational project with the main goal of obtaining CCD photometric observations of selected minor planets (Sárneczky et al. 1999; Kiss et al. 1999). This paper reports new observations of NEOs carried out at three observatories, between February, 1999 and July, 2000. The data acquisition, main geometric parameters and applied methods are described in Sect. 2, while Sect. 3 deals with the detailed observational results.

\section{Observations and modeling methods}

\subsection{Data acquisition}

Observations have been made at three different observing sites. Johnson $V$ and $R_{\mathrm{C}}$ filtered CCD observations were carried out at Calar Alto Observatory (Spain) on 10 nights in June-July, 2000 (we also observed distant active comets during this observing run - see Szabó et al. 2001). The instrument used was the $123 / 981 \mathrm{~cm}$ Cassegrain telescope (hereafter designed by [1]) equipped with the SITe\#2b CCD camera $(2048 \times 2048$ pixels giving an angular unbinned resolution of $0.49 / \mathrm{pixel})$. The projected sky area is $17 !^{\prime} 0 \times 17^{\prime} .0,10^{\prime} .0 \times 10^{\prime} .0$ unvignetted.

We carried out $R_{\mathrm{C}}$ filtered CCD observations at Piszkéstető Station of Konkoly Observatory on two nights in September, 1999 and on one night in January, 2000. The data were obtained using the 60/90/180 cm 
Schmidt-telescope [2] equipped with a Photometrics AT200 CCD camera $(1536 \times 1024 \mathrm{KAF} 1600$ MCII coated CCD chip). The projected sky area is $29^{\prime} \times 18^{\prime}$ which corresponds to an angular resolution of 1 !' $1 /$ pixel. The photometric effect of neglecting standard transformation was discussed in Kiss et al. (1999), where we illustrated the usability of single-filtered data.

R-filtered and unfiltered CCD photometry was carried out on two nights at the University of Szeged using a 0.28-m Schmidt-Cassegrain telescope [3] located in the very center of the city of Szeged. Two detectors were used. The SBIG ST-6 CCD camera $(375 \times 242$ pixels $)$ gives an angular resolution of about $2^{\prime \prime} /$ pixel (the pixels are rectangular). The second detector was an SBIG ST-7 CCD camera $(765 \times 510$ pixels $)$ giving an angular resolution of about $1^{\prime \prime} 0 /$ pixel. The pixels are also rectangular.

The instruments used together with the times of exposure are summarized in Table 1. The last column gives the comparison stars. In the case of relative photometry the local comparison stars in the fields are identified by their GSC 1.2 (Guide CD-ROM Star Chart 1997) or USNO A2.0 (Monet et al. 1998) numbers and magnitudes. When absolute photometry was done (at Calar Alto Observatory), the standard fields of Landolt (1992) are included. Because of the fast motion of 1999 CV3, the apparent path of the asteroid could be covered only by three overlapping frames, therefore, three different comparison stars were used on the same night. The aspect data of the observations are presented in Table 2 .

The exposure times were limited by two factors: firstly, the asteroids were not allowed to move more than the $F W H M$ of the stellar profiles (varying from night to night) and secondly, the signal-to-noise $(S N)$ ratio had to be at least 10. This latter parameter was estimated by comparing the peak pixel values with the sky background during the observations.

Selecting the appropriate size of the photometric aperture is of considerable importance when doing aperturephotometry of faint objects with remarkable fast motion. Selecting too small apertures causes loss of light, while selecting too large radii increases the noise in the aperture. Usually, observers select a series of apertures during the reduction, and the "best" lightcurve is accepted. In our analysis a different approach has been used. As the elongated profiles of the moving asteroids can be hardly treated analytically, we have performed a semi-experimental optimalization of the signal-to-noise ratio based on the photometric behaviour of the profiles.

Assuming a homogeneous noise model with the background scatter density value (scatter/picture element) $n$, the square of the total noise $N$ present in the aperture with a radius of $R$ can be taken as the sum of the noise in every picture element:

$N^{2}=n^{2}\left(R^{2} \pi\right)=(1.772 R \cdot n)^{2}$.

Let $S$ be the measured signal. The aperture radius with the highest $S / N$ ratio is established by taking its differential

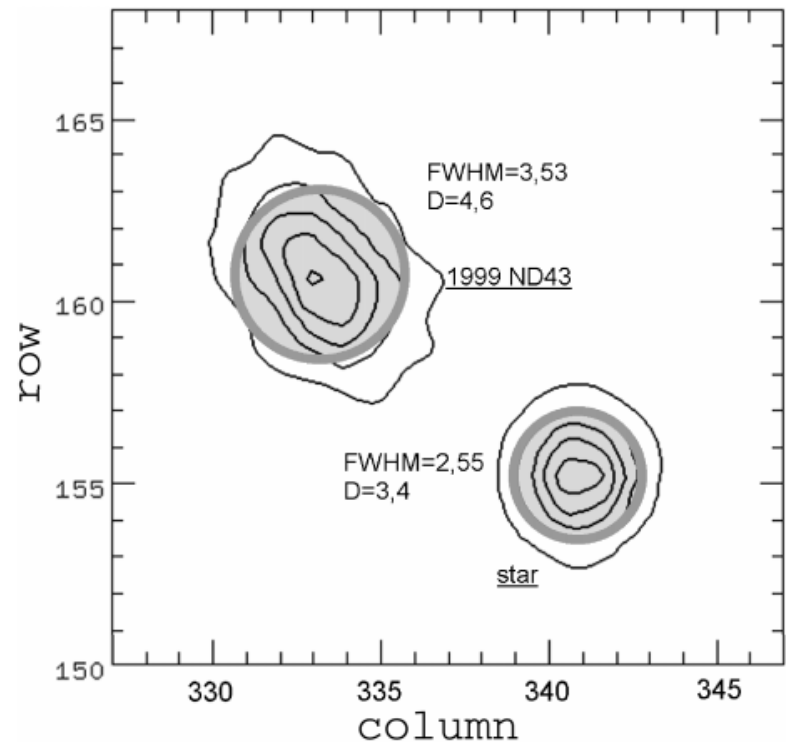

Fig. 1. Profiles of a star and an asteroid fitted with the optimal aperture of the reduction (see text for details).

$$
\begin{aligned}
\mathrm{d} \frac{S}{N} & =\frac{N \cdot \mathrm{d} S-S \cdot \mathrm{d} N}{N^{2}}=1.772 n \frac{R \cdot \mathrm{d} S-S \cdot \mathrm{d} R}{(1.772 \cdot R)^{2}} \\
& =\frac{n}{1.772 R^{2}}(R \cdot \mathrm{d} S-S \cdot \mathrm{d} R)=0 .
\end{aligned}
$$

As the whole expression equals 0 for the optimal $S / N$, one can divide the equation by $\frac{n}{1.772 R^{2}}$. Elimination of $n$ yields to the fundamental conclusion that the diameter of the optimal aperture does not depend on the scatter of the background. This is true for all circular and elongated profiles on the assumption of homogeneous noise.

After rearranging Eq. (2) one gets $R \mathrm{~d} S=S \mathrm{~d} R$, the solution of which is:

$\ln R=\ln S=\ln 10^{-0.4 *\left(m-m_{0}\right)}=-0.92\left(m-m_{0}\right)$,

where $m$ is the measured brightness in the aperture, expressed in magnitudes, while $m_{0}$ is the integration constant. In an aperture of radius $R+\Delta R$ the measured magnitude is $m-\Delta m$. If $\Delta m$ is small, the following expression will be also true for the increased aperture:

$\ln (R+\Delta R)=-0.92\left(m-m_{0}-\Delta m\right)$.

Taking the difference (4)-(3) and using $\ln (R+\Delta R)-$ $\ln (R)=\Delta R / R$, we get the final expression valid for the optimal aperture:

$\frac{\Delta R}{R}=0.92 \Delta m$

The left-hand side expresses the relative increment of the radius, while the right-hand side is the corresponding magnitude change. In practice, the radius of the aperture is altered and the alteration of the measured magnitude is examined. When their values satisfy Eq. (5), the aperture is accepted to be the optimal one.

The projection of the calculated aperture onto the stellar and asteroidal profiles is presented in Fig. 1. We note 
Table 1. Data of the used instruments and comparison stars. Subscripts "i" and "s" denote whether the data are in instrumental or standard photometric system. See text for telescope codes.

\begin{tabular}{|c|c|c|c|c|c|}
\hline Date & Telescope & Detector & Filter & $\operatorname{Exp}(\mathrm{s})$ & Comp. \\
\hline \multicolumn{6}{|l|}{699 Hela } \\
\hline 199909 15/16 & {$[3]$} & ST7 & $R_{\mathrm{i}}$ & 50 & GSC 27341730 \\
\hline \multicolumn{6}{|l|}{1865 Cerberus } \\
\hline $19990924 / 25$ & {$[2]$} & AT200 & $R_{\mathrm{i}}$ & 60 & USNO $012243+254115$ \\
\hline $20000708 / 09$ & {$[1]$} & $\mathrm{SITe} \# 2 \mathrm{~b}$ & $R_{\mathrm{S}}$ & 240 & PG $1633+099$ \\
\hline $20000709 / 10$ & {$[1]$} & $\mathrm{SITe} \# 2 \mathrm{~b}$ & $R_{\mathrm{S}}$ & 240 & PG $1633+099$ \\
\hline $20000710 / 11$ & {$[1]$} & SITe\#2b & $R_{\mathrm{S}}$ & 240 & PG $1633+099$ \\
\hline \multicolumn{6}{|l|}{1866 Sisyphus } \\
\hline $\begin{array}{l}20000630 / 0701 \\
(\mathbf{1 1 4 0 5 )} \mathbf{1 9 9 9} \mathbf{C V 3}\end{array}$ & {$[1]$} & SITe\#2b & $R_{\mathrm{s}}$ & 240 & PG 1525-071 \\
\hline 19990227.8 & {$[3]$} & ST6 & unf & 30 & GSC 3434172 \\
\hline 19990227.9 & {$[3]$} & ST6 & unf & 30 & GSC 3434144 \\
\hline $\begin{array}{l}199902 \quad 28.0 \\
(\mathbf{1 6 0 6 4 )} \mathbf{1 9 9 9} \mathbf{R H 2 7}\end{array}$ & {$[3]$} & ST6 & unf & 30 & GSC 343496 \\
\hline $20000101 / 02$ & {$[2]$} & AT200 & $R_{\mathrm{i}}$ & 60 & USNO $043039+395018$ \\
\hline \multicolumn{6}{|l|}{1999 JD6 } \\
\hline $20000702 / 03$ & {$[1]$} & SITe\#2b & $R_{\mathrm{S}}$ & 60 & PG $1633+099$ \\
\hline $20000705 / 06$ & {$[1]$} & SITe\#2b & $R_{\mathrm{S}}$ & 60 & PG $1633+099$ \\
\hline \multicolumn{6}{|l|}{1999 ND43 } \\
\hline $19990923 / 24$ & {$[2]$} & AT200 & $R_{\mathrm{i}}$ & 60 & GSC 3302711 \\
\hline \multicolumn{6}{|l|}{2000 GK137 } \\
\hline $20000629 / 30$ & {$[1]$} & $\mathrm{SITe} \# 2 \mathrm{~b}$ & $R_{\mathrm{s}}$ & 60 & PG $1657+078$ \\
\hline 200007 01/02 & {$[1]$} & SITe\#2b & $R_{\mathrm{s}}$ & 60 & PG $1657+078$ \\
\hline \multicolumn{6}{|l|}{$2000 \mathrm{NM}$} \\
\hline 200007 03/04 & {$[1]$} & $\mathrm{SITe} \# 2 \mathrm{~b}$ & $R_{\mathrm{S}}$ & 20 & S.Area 110 \\
\hline $20000704 / 05$ & {$[1]$} & SITe\#2b & $R_{\mathrm{s}}$ & 20 & S.Area 110 \\
\hline $20000705 / 06$ & {$[1]$} & SITe\#2b & $R_{\mathrm{S}}$ & 20 & S.Area 110 \\
\hline
\end{tabular}

that the fit of the circular aperture with the elongated profile is not very good, which decreases the attainable $S / N$ ratio. The optimal aperture radius depends slightly on the apparent motion, its average size is about 130 percent of the FWHM.

The image reduction was done with standard IRAF routines. Aperture photometry was performed with the IRAF task noao.digiphot.apphot.qphot. Two nearby comparison stars were selected and their differential light curves were examined to estimate the photometric accuracy. The scatter arising from the comparison stars was estimated to be $\pm 0{ }^{\mathrm{m}} 01-0$. 03 , depending on the weather conditions. (We note, that our time series observations were also analysed by Csák et al. 2000 resulting in the discovery of 13 new short period variable stars. Therefore, we could solidly exclude variables from the chosen comparison stars.) The photometric stability indicated by the relatively low scatter of the brightness differences of the comparison stars suggests that the motion and the faintness of the asteroid is the dominating factor for the higher scatter of the lightcurves. Thus, the above described optimalization procedure was applied for the detected asteroid profiles resulting in a slightly larger aperture radius than that for the stellar profiles, namely $1.3 \times F W H M$. The comparison stars were, of course, measured with the same aperture.
In the case of the instrumental lightcurves, the presented magnitudes throughout the paper are based on magnitudes of the comparison stars taken from the Guide Star Catalogue (GSC) (Table 1). Therefore, their absolute values are fairly uncertain (at a level of $\pm 0.2-0.3$ ). Fortunately, the shape of the lightcurve is not affected by neglecting the standard transformations.

The sky conditions at Calar Alto Observatory allowed doing standard all-sky photometry. The standard sequences were selected from the list of Landolt (1992), containing 4-5 standard stars covering $V$ magnitudes between 13-15 magnitudes and $V-R$ indices between -0 . 1 and 0.6 . In order to monitor the extinction correction, the fields were observed in every hour.

The presented data were corrected for light-travel time and are available electronically at CDS-Strasbourg.

\subsection{Methods of analysis}

The main aim of our observations was to get information on the rotational states of NEOs. The period of rotation can be inferred when more than one rotational phase is covered. Determination the sense of rotation requires a more complex analysis of lightcurves taken at different ecliptic positions. As there have been sufficient data series 
Table 2. The journal of observations. $(r-$ heliocentric distance; $\Delta$ - geocentric distance; $\lambda$ - ecliptic longitude; $\beta$ - ecliptic latitude; $\alpha$ - solar phase angle; aspect data are referred to 2000.0).

\begin{tabular}{|c|c|c|c|c|c|c|c|}
\hline Date & RA & Decl. & $r(\mathrm{AU})$ & $\Delta(\mathrm{AU})$ & $\lambda\left({ }^{\circ}\right)$ & $\beta\left(^{\circ}\right)$ & $\alpha\left(^{\circ}\right)$ \\
\hline \multicolumn{8}{|l|}{699 Hela } \\
\hline 199909 15/16 & 2228 & +3056 & 1.57 & 0.65 & 327 & 20 & 23 \\
\hline \multicolumn{8}{|l|}{1865 Cerberus } \\
\hline $19990924 / 25$ & 0122 & +2538 & 1.55 & 0.61 & 28 & 16 & 20 \\
\hline 200007 08/09 & 0011 & +2741 & 1.37 & 0.87 & 9 & 28 & 48 \\
\hline $20000709 / 10$ & 0011 & +2759 & 1.37 & 0.87 & 9 & 28 & 48 \\
\hline 200007 10/11 & 0012 & +2805 & 1.38 & 0.86 & 10 & 28 & 47 \\
\hline \multicolumn{8}{|l|}{1866 Sisyphus } \\
\hline $\begin{array}{l}20000630 / 0701 \\
(\mathbf{1 1 4 0 5}) \mathbf{1 9 9 9} \mathbf{C V 3}\end{array}$ & 1249 & 2657 & 2.73 & 2.69 & 195 & 20 & 22 \\
\hline 19990227.8 & 1000 & +4648 & 1.16 & 0.22 & 168 & 53 & 32.7 \\
\hline 19990227.9 & 1000 & +4702 & 1.16 & 0.22 & 168 & 53 & 33.0 \\
\hline 19990228.0 & 1000 & +4715 & 1.16 & 0.22 & 168 & 53 & 33.2 \\
\hline \multicolumn{8}{|l|}{ (16064) 1999 RH27 } \\
\hline $20000101 / 02$ & 0431 & +3948 & 1.24 & 0.29 & 54 & 61 & 26 \\
\hline \multicolumn{8}{|l|}{1999 JD6 } \\
\hline 200007 02/03 & 1748 & +1030 & 1.34 & 0.38 & 263 & -13 & 28 \\
\hline 200007 05/06 & 1733 & +1045 & 1.32 & 0.37 & 261 & -13 & 30 \\
\hline \multicolumn{8}{|l|}{1999 ND43 } \\
\hline $19990923 / 24$ & 0217 & +4921 & 1.06 & 0.10 & 50 & 33 & 53 \\
\hline \multicolumn{8}{|l|}{2000 GK137 } \\
\hline $20000629 / 30$ & 1955 & +5833 & 1.05 & 0.17 & 287 & 37 & 74 \\
\hline 200007 01/02 & 2019 & +6034 & 1.04 & 0.17 & 294 & 39 & 77 \\
\hline \multicolumn{8}{|l|}{2000 NM } \\
\hline 200007 03/04 & 1809 & -0506 & 1.17 & 0.16 & 272 & -5 & 18 \\
\hline $20000704 / 05$ & 1807 & -0159 & 1.16 & 0.16 & 272 & -2 & 21 \\
\hline 200007 05/06 & 1804 & +0121 & 1.15 & 0.15 & 272 & 0 & 24 \\
\hline
\end{tabular}

in the literature for 1865 Cerberus, a rotation model could be also calculated.

Two main types of method are used widely. The amplitude-method was described, e.g., by Magnusson (1989). For this, the amplitude information is used to determine the spin vector and the shape. An important point is that the observed $A(\alpha)$ amplitudes at solar phase $\alpha$ should be reduced to zero phase $\left(A\left(0^{\circ}\right)\right)$, if possible, by a simple linear transformation in form of $A(\alpha)=A\left(0^{\circ}\right)(1+m \alpha)$.

The other possibility is to examine the times of light extrema ("epoch-methods", "E-methods", see, e.g., Magnusson 1989; Detal et al. 1994). In this paper a modified version is used by constructing the $\mathrm{O}-\mathrm{C}$-ecliptic longitude diagrams as a function of revolution. Examining the virtual phase shifts ("observed minus calculated") occurring during a revolution helps to establish the sidereal period of rotation. The trend of the phase shifts gives the sense of rotation. Increasing $\mathrm{O}-\mathrm{C}$ ' (i.e. phase shift) refers to prograde rotation, while retrograde rotators have decreasing $\mathrm{O}-\mathrm{C}$ '. The method is briefly outlined in Kiss et al. (1999) and in Szabó et al. (1999), and will be thor- oughly presented and discussed in a forthcoming paper (Szabó et al., in prep.).

\section{Discussion}

In the following, we describe the observational details and results.

\section{Hela}

This asteroid was discovered by J. Helffric in Heidelberg, in 1910. Although this unusual object does not belong to the real Earth-grazing asteroids, it can pass quite close to the Earth in perihelion $(q=1.55 \mathrm{AU})$. The first photometry was done by Binzel (1987), who presented nine data points. He obtained a period of about 3 hours, while the measured amplitude was over 0.53 at the ecliptic position $\lambda=300^{\circ}$ and $\beta=18.8$ (the solar phase angle was $\left.\alpha=23^{\circ}\right)$.

Our observations carried out with instrument [3] on September 15, 1999 were done very close to the perihelion passage. The amplitude of 0.18 turned out to be smaller than the one previously detected. As the solar phase angle is similar for the two sets of observations (ours and 
699 Hela

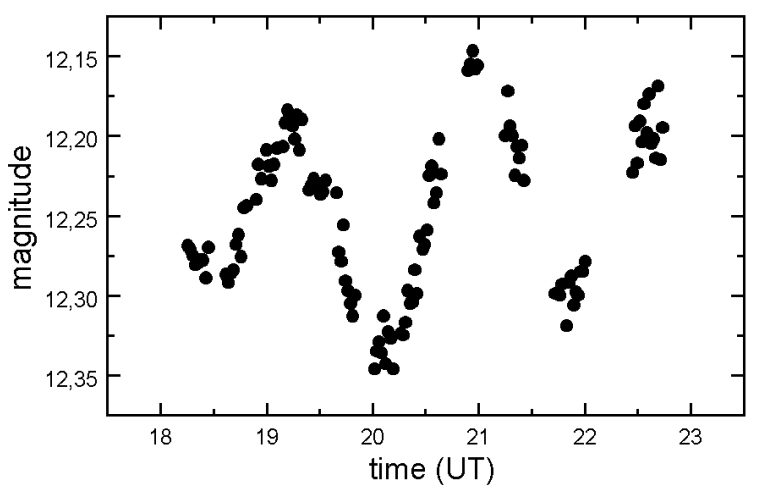

Fig. 2. The $R_{\mathrm{C}}$ lightcurve of 699 on September 15th, 1999.

1865 Cerberus

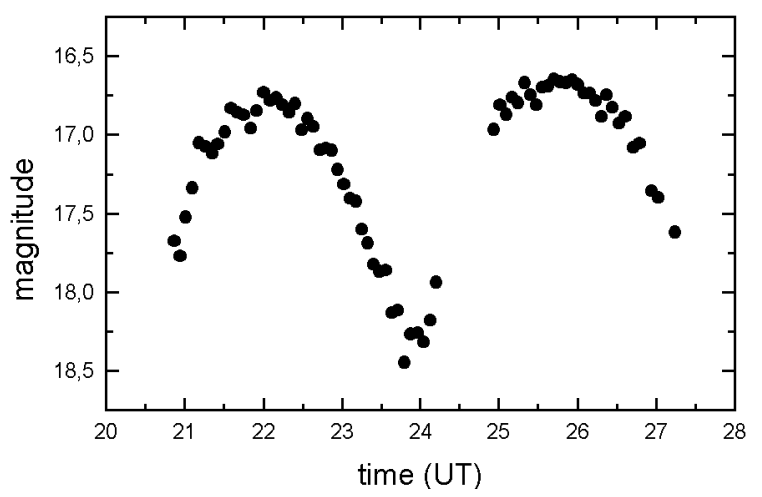

Fig. 3. The instrumental $R_{\mathrm{C}}$ lightcurve of 1865 on September 24th, 1999.

that of Binzel 1987), the significant difference of the amplitudes cannot be the effect of the varying aspect angle. The most likely value for the synodic period is $3.3 \pm 0.2$. The lightcurve is presented in Fig. 2.

\section{Cerberus}

This asteroid has one of the largest observed amplitudes of all minor planets. The first photometry including 41 data points was discussed in Harris \& Young (1989); later, Wisniewski et al. (1997) presented the second lightcurve of this asteroid. We presented the lightcurve in the 1998 opposition in Sárneczky et al. (1999). In this paper both the lightcurves of the opposition in 1999 (Fig. 3) and the composite lightcurve observed between 08/09-10/11 July (Fig. 4), 2000 are presented. Measured amplitudes $\left(A_{\text {obs }}\right)$ and times of extrema $\left(t_{\min }\right)$ at different aspects are summarized in Table 3.

Based on earlier data (see Table 3), we tried to determine a new model by the amplitude method. The $m$ parameter was estimated by minimizing the scatter of the longitude-amplitude diagram, resulting $m=$ $0.018 \pm 0.005$.

The O-C' model has been determined (Fig. 5). Because of the distribution of data, the spin axis cannot be estimated based on the $\mathrm{O}-\mathrm{C}$ ' points. The ecliptic position was almost the same at the time of the obser-

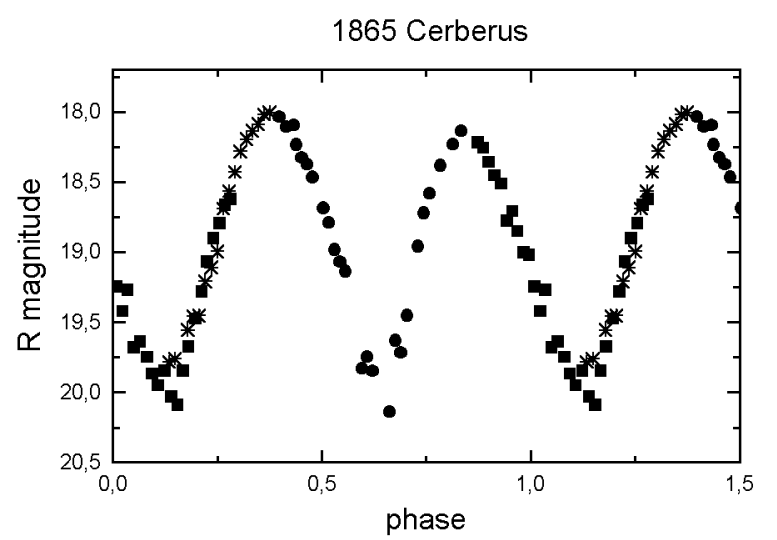

Fig. 4. The composite $R_{\mathrm{C}}$ lightcurve of 1865 in July, 2000. Data on the individual nights are denoted by solid squares (07.08), solid circles (07.09) and asterisks (07.10). Zero phase is at JD 2451734.490.

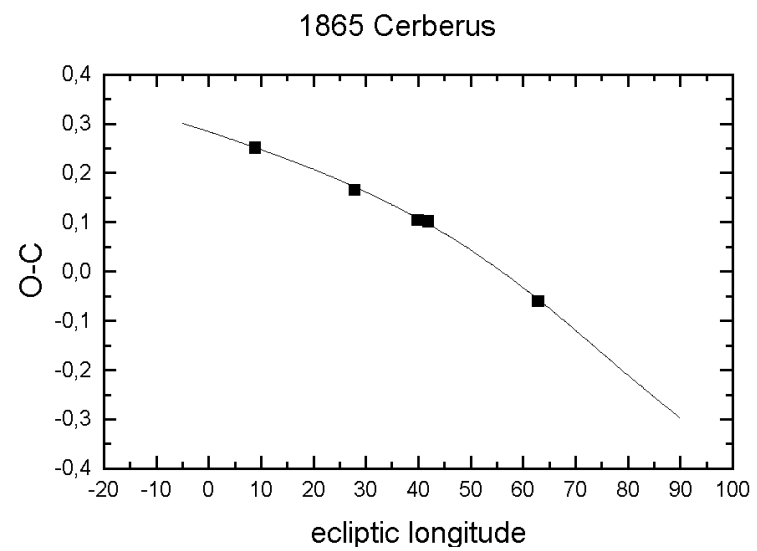

Fig. 5. The observed $\mathrm{O}-\mathrm{C}$ ' values fitted with the model for 1865 Cerberus.

vations in 1980 and 1988, though the observations were made many years apart. This has quite a favourable influence on the calculations of the sidereal period. A value of $P_{\text {sid }}=0.27024003 \pm 0$ d 00000005 was found. The O-C' graph decreases which indicates retrograde rotation.

\section{Sisyphus}

This asteroid was discovered by P. Wild using a $40 \mathrm{~cm}$ Schmidt-telescope in 1972. The first photometry was presented by Schober et al. (1993).

During our observations the previously measured period of 2.7 hours was confirmed. The amplitude was $0 .{ }^{\mathrm{m}} 12$ close to the previously determined 0.13 (Schober et al. 1993). During the observations the seeing was about 0 ". 7 at the altitude of the asteroid. This circumstance had a fortunate influence on the data reduction, although the apparent motion was about 1 .' 6 during the 4 min of integration. The lightcurve is presented in Fig. 6.

\section{(11405) 1999 CV3}

The asteroid was discovered by the LINEAR project in Socorro. Thanks to its close path to the Earth its maximum brightness was about $13 \mathrm{mag}$ on the night of the observation. Its proper motion was quite fast with a value 
Table 3. Published photometries of 1865 Cerberus.

\begin{tabular}{lrrrlll}
\hline Date & $\lambda\left({ }^{\circ}\right)$ & $\beta\left(^{\circ}\right)$ & $\alpha\left(^{\circ}\right)$ & $A_{\text {obs }}$ & $t_{\min }$ & ref. \\
\hline $19801104-06$ & 40 & -12 & 10 & $1^{\mathrm{m}} 45$ & 2444548.979 & $(1)$ \\
$19891103-04$ & 63 & -17 & 22 & 1.8 & 2447835.76245 & $(2)$ \\
$1998 \quad 1023,26$ & 42 & -2.8 & 38.7 & 2.3 & 2451113.515 & $(3)$ \\
19990924 & 28 & 16 & 20 & 1.7 & 2451446.455 & $(4)$ \\
$2000 \quad 0708-10$ & 9 & 28 & 48 & 2.2 & 2451734.536 & $(4)$ \\
\hline
\end{tabular}

References: (1) - Harris \& Young (1989); (2) - Wisniewski et al. (1997); (3) - Sárneczky et al. (1999); (4) - present paper.

1866 Sisyphus

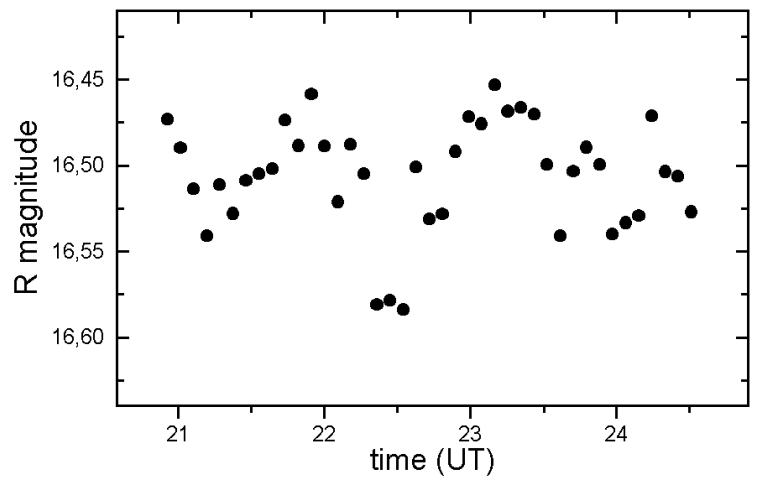

Fig. 6. The $R_{\mathrm{C}}$ lightcurve of 1866 on June $30 \mathrm{th}, 2000$.

(11405) $1999 \mathrm{CV} 3$

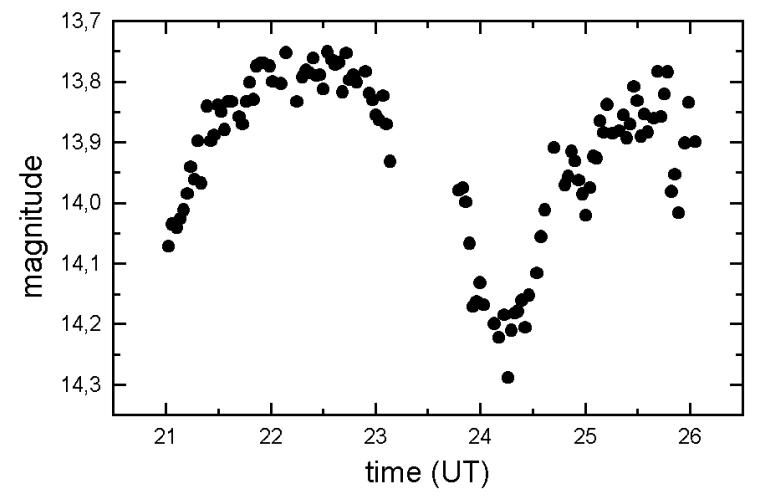

Fig. 7. The unfiltered lightcurve of 1999 CV3 on February 27th, 1999.

of $360^{\prime \prime}$ / hour, which is why we had to use 3 different comparison stars located on 3 overlapping star fields along the path during the 5 hours of observation. The fast motion did not permit exposure times of over $20 \mathrm{~s}$.

Its period is longer than the observing interval but assuming a simple "double" lightcurve it may be about 7 hours. The measured amplitude is 0.43 . The lightcurve is presented in Fig. 7.

\section{(16064) 1999 RH27}

This Earth-grazing asteroid was discovered by the Catalina Sky Survey on 5th September, 1999. During our observations at Konkoly Observatory, a quite ambiguous light variation was detected. During the 3 hours of observation the lightcurve showed a decrease of 0.1 . The am-

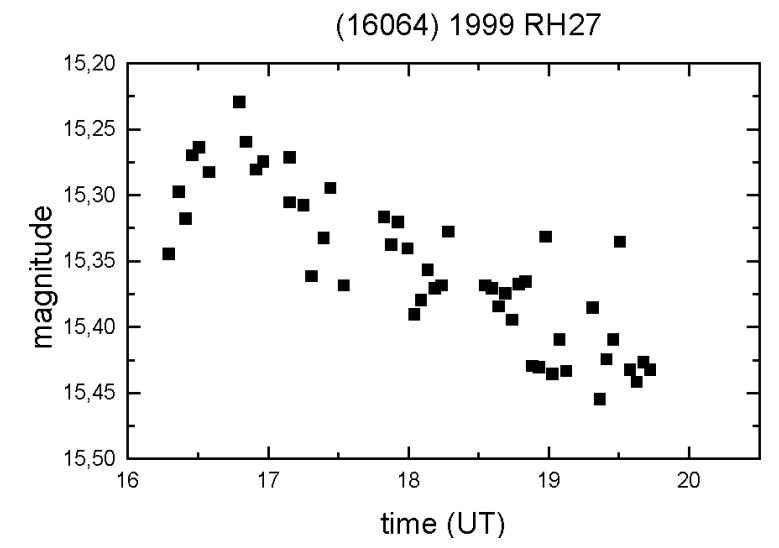

Fig. 8. The $R_{\mathrm{C}}$ lightcurve of 1999 RH27 on January 1st, 2000.

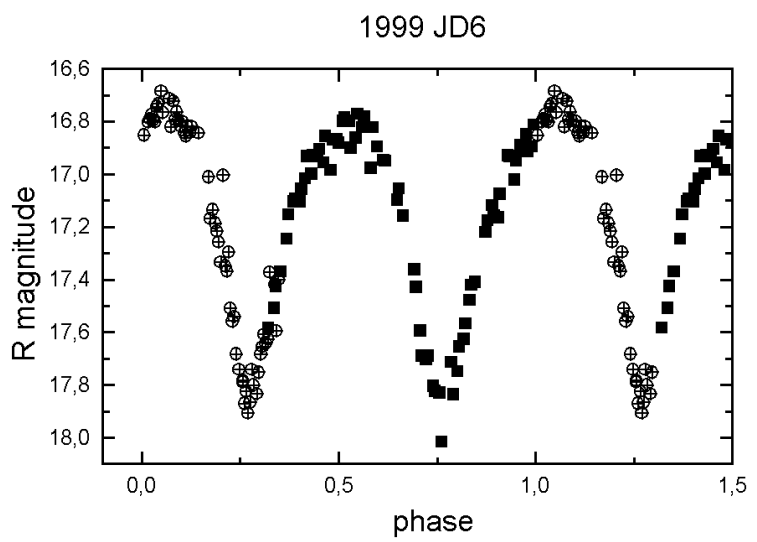

Fig. 9. Composite lightcurve of 1999 JD6. Data series of the individual nights are denoted by crossed circles (07.02) and solid squares (07.05). Zero phase is at 2451728.38408.

plitude is higher than this value while the period seems to be much longer than the observing run. The lightcurve is presented in Fig. 8.

\section{JD6}

This member of the Aten family was discovered by the LONEOS project in Flagstaff. The equipment used was a $59 \mathrm{~cm}$ Schmidt-telescope. It turned out to be another high-amplitude NEO with a value of $1.2 \pm 0.1$ magnitude. The period of the light variation is $7.68 \pm 0.03$ hours. The lightcurve is quite similar to the other high-amplitude asteroids, e.g. 1865 Cerberus as the maxima take much more time than the sharp minima. That can be explained by the rotation of a splinter-like body. The composite lightcurve is presented in Fig. 9. 


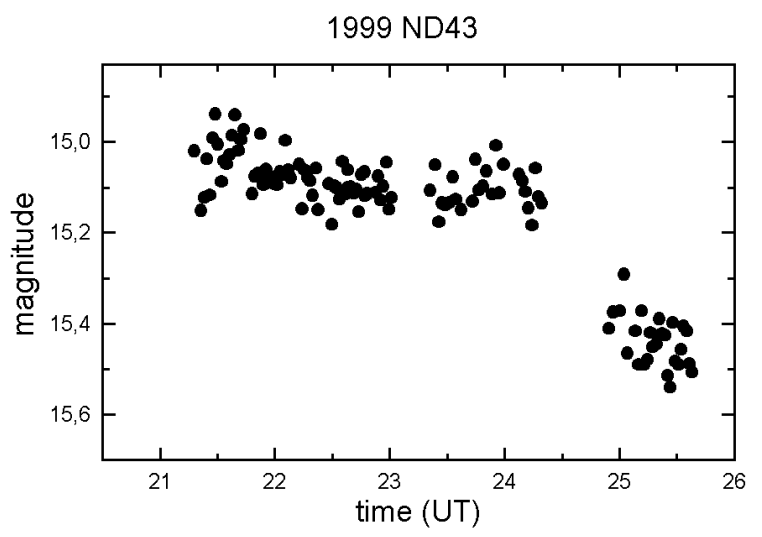

Fig. 10. The $R_{\mathrm{C}}$ lightcurve of 1999 ND43 on September 23th, 1999.

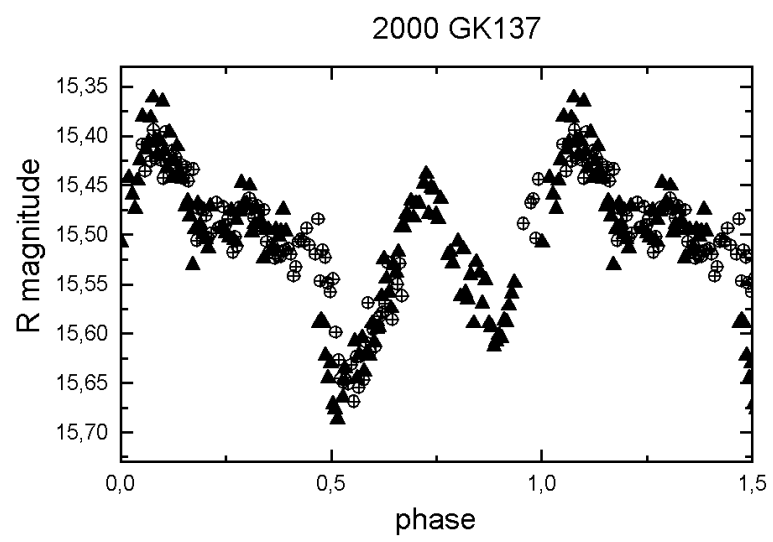

Fig. 11. Composite lightcurve of 2000 GK137. Data series of the individual nights are denoted by crossed circles (06.29) and up triangles (07.01). Zero phase is at 2451727.37817.

\section{ND43}

This asteroid was discovered also by LINEAR in Socorro, on July 14th, 1999. The instrument used was the $1 \mathrm{~m}$ reflector.

During the observations the asteroid was in a distance of $0.10 \mathrm{AU}$. Its apparent motion was almost $3^{\prime \prime} / \mathrm{min}$, but because of its faintness we had to increase the exposure time up to $1 \mathrm{~min}$. The highly elongated profile was presented in Fig. 1 in Sect. 2. The 5 hours-long lightcurve does not show periodic light variation, only an obvious faintening of about 0.5 is visible, as presented in Fig. 10.

\section{GK137}

This NEO was discovered by the LINEAR project in Socorro. The object was included in our target list following the suggestions of P. Pravec (personal communication). In the present paper, data obtained on two nights are presented. The composite lightcurve was calculated using a period of $4.84 \pm 0.02$. The shape of the lightcurve is quite asymmetric, the brighter maximum is followed by a nearly constant segment. Considering the high solar phase during the observing run $\left(\alpha \approx 75^{\circ}\right)$, the lightcurve is likely to simply reflect local surface irregularities. The total amplitude of the lightcurve is 0.27 .
2000 NM

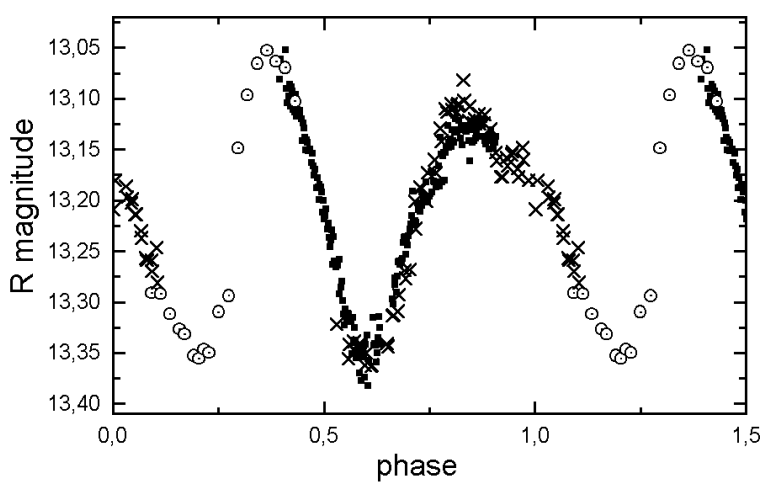

Fig. 12. The composite lightcurve of 2000 NM. Data series of the individual nights are denoted by solid squares (07.03), dotted circles (07.04) and $\times(07.05)$. Zero phase is at 2451731.50942 .

Table 4. The determined periods, amplitudes, spin vectors and shapes.

\begin{tabular}{llll}
\hline Asteroid & $P_{\text {syn }}(\mathrm{h})$ & $P_{\text {sid }}(\mathrm{d})$ & $A_{\text {obs }}(\mathrm{mag})$ \\
\hline 699 & 3.3 & & 0.18 \\
1865 & & 0.27024003 & \\
1866 & 2.7 & & 0.12 \\
11405 & $>5$ & & 0.43 \\
16064 & $>3$ & & $>0.1$ \\
1999 JD6 & 7.68 & & 1.2 \\
1999 ND43 & $>5$ & & $>0.5$ \\
2000 GK137 & 4.84 & & 0.27 \\
2000 NM & 9.24 & & 0.30 \\
\hline
\end{tabular}

\section{$2000 N M$}

This asteroid was discovered by amateur astronomer L. L. Amburgey on July 2.17 UT, 2000. The instrument used was a $21 \mathrm{~cm}$ telescope and the asteroid was found on CCD images at a brightness of 13 magnitudes. 39 hours after its discovery we observed the light variation. Because of the fast motion the exposure time had to be decreased to $20 \mathrm{~s}$ which was also permitted by the high brightness of the asteroid. Based on the observations obtained on three nights the period has been determined to be $9.24 \pm 0.02$, while the amplitude is 0.30 . The composite diagram is presented in Fig. 12.

The lightcurve has three humps similar to 2000 GK137. The ratio between the shorter and longer hump is $1: 2$, but contrary to 2000 GK137, the shorter maximum has the brighter maximal brightness. The irregularities in the lightcurve are quite surprising as they cannot be explained by the effect of the high solar phase (see Table 1 and the discussion of 2000 GK137).

We summarize the resulting synodic periods, amplitudes and models in Table 4.

Acknowledgements. This research was supported by the FKFP Grant 0010/2001, Pro Renovanda Cultura Hungariae Grants DT 2000. máj./43., DT 2000. máj./44., DT 2000. máj./48. and DT 1999. ápr./23, OTKA Grant \#T032258, "Bolyai János" Research Scholarship of LLK from the Hungarian Academy 
of Sciences and Szeged Observatory Foundation. The warm hospitality and helpful assistance of the staff of Calar Alto Observatory and Konkoly Observatory and their provision of telescope time is gratefully acknowledged. The NASA ADS Abstract Service was used to access data and references.

\section{References}

Binzel, R. P. 1985, Icarus, 63, 99

Binzel, R. P. 1987, Icarus, 72, 135

Binzel, R. P. 2000, P\&SS, 48, 297

Csák, B., Kiss, L. L., Szabó, Gy., et al. 2000, IBVS, No. 4989

Detal, A., Hainaut, O., Pospieszalska-Surdej, A., et al. 1994, A\&A, 281, 269

Guide CD-ROM Star Chart 1997, Project Pluto, http://www.projectpluto.com

Harris, A. W., \& Young, J. W. 1989, Icarus, 81, 314

Kiss, L. L., Szabó, Gy., \& Sárneczky, K. 1999, A\&AS, 140, 21

Landolt, A. U. 1992, AJ, 104, 340
Magnusson, P. 1989, in Asteroids II (Univ. of Arizona Press), 1180

Monet, D., et al. 1998, A catalogue of astrometric standards, The PMM USNO-A2.0 Catalog

Pravec, P., Sarounová, L., \& Rabinowitz, D. L. 2000, Icarus, 146, 190

Pravdo, S. H., Rabinowitz, D. L., Helin, E. F., et al. 1999, AJ, 119,1616

Sárneczky, K., Szabó, Gy., \& Kiss, L. L. 1999, A\&AS, 137, 363

Schober, H. J., Erikson, A., Hahn, G., \& Lagerkvist, C.-I. 1993, A\&AS, 101, 499

Steel, D. I., \& Marsden, B. G. 1996, EM\&P, 74, 85

Stokes, G. H., Evans, J. B., Viggh, H. E., et al. 2000, Icarus, 148, 21

Szabó, Gy., Sárneczky, K., \& Kiss, L. L. 1999, Proc. of IAU Coll., 173, 185

Szabó, Gy., Csák, B., Sárneczky, K., \& Kiss, L. L. 2001, A\&A, 374,712

Wisniewski, W. Z., Michałowski, T. M., Harris, A. W., \& McMillan, R. S. 1997, Icarus, 126, 395 